\title{
Restricted domains with Pareto free pairs
}

Citation for published version (APA):

Storcken, T. (2021). Restricted domains with Pareto free pairs. Maastricht University, Graduate School of Business and Economics. GSBE Research Memoranda No. 012 https://doi.org/10.26481/umagsb.2021012

Document status and date:

Published: 16/09/2021

DOI:

10.26481/umagsb.2021012

Document Version:

Publisher's PDF, also known as Version of record

\section{Please check the document version of this publication:}

- A submitted manuscript is the version of the article upon submission and before peer-review. There can be important differences between the submitted version and the official published version of record.

People interested in the research are advised to contact the author for the final version of the publication, or visit the DOI to the publisher's website.

- The final author version and the galley proof are versions of the publication after peer review.

- The final published version features the final layout of the paper including the volume, issue and page numbers.

Link to publication

\footnotetext{
General rights rights.

- You may freely distribute the URL identifying the publication in the public portal. please follow below link for the End User Agreement:

www.umlib.nl/taverne-license

Take down policy

If you believe that this document breaches copyright please contact us at:

repository@maastrichtuniversity.nl

providing details and we will investigate your claim.
}

Copyright and moral rights for the publications made accessible in the public portal are retained by the authors and/or other copyright owners and it is a condition of accessing publications that users recognise and abide by the legal requirements associated with these

- Users may download and print one copy of any publication from the public portal for the purpose of private study or research.

- You may not further distribute the material or use it for any profit-making activity or commercial gain

If the publication is distributed under the terms of Article $25 \mathrm{fa}$ of the Dutch Copyright Act, indicated by the "Taverne" license above, 


\section{Maastricht University}

Ton Storcken

Restricted domains with Pareto free pairs

$\mathrm{RM} / 21 / 012$

ISSN: $2666-8807$

\section{GSBE}

Maastricht University School of Business and Economics

Graduate School of Business and Economics

P.O Box 616

NL-6200 MD Maastricht

The Netherlands 


\title{
Restricted domains with Pareto free pairs
}

\author{
Ton Storcken*
}

June 2021

\begin{abstract}
Among the domains restricted by Pareto free pairs we determine those allowing for preference rules being anonymous and independent of irrelevant alternatives. Essentially such preference rules appear to be based on a priority ordered at which adjacent alternatives can only be swapped in order is all agents aggree with this swap.
\end{abstract}

\section{Introduction}

As the impossibility theorems such as of Arrow[1978], Gibbard[1973], and Satterthwaite[1975] do not reflect our daily life experience on collective decision making, relaxations of the conditions imposed on their models have been studied intensively. Restricting the domain of the collective decision rules is one of the possible options. Indeed, the unrestricted domain assumption means that the collective decision rule should assign an outcome to every potentially possible profile, i.e. a combination of individual preferences. Forcing the collective decision rule to produce an outcome at some profiles may via its imposed properties fix the outcome at other profiles and therewith narrow down the possible set of decision rules satisfying all model conditions. Often, this boils down to dictatorial collective decision rules being the only decision rules satisfying the model conditions. In that view restricting the domain of a collective decision rule may enforced less outcome correlations. It therewith may allow for more collective decision rules, for instance anonymous rules.

Pairwise majority rules appear to be possible whenever the profile is "single peaked". That is, with respect to some natural ordering the alternatives can be identified with numbers on the real line and agents' preferences can be represented by unimodal utility functions. See among many others Black[1948] and Moulin[1980]. These results are fruitful and help to design collective decision making rules. But, the likeliness of a "single peaked" profile is limited.

Here we study domain restrictions by assuming the absence of global consensus between at least some pairs of alternatives. For cases with a great number of agents, such as at country wide elections, one might expect that not every

*Maastricht University, corresponding e-mail address: t.storcken@maastrichtuniversity.nl 
agent prefers alternative $a$ above alternative $b$. We investigate which Pareto optimal and anonymous preference rules are independent of irrelevant alternatives at domains when some pairs of alternatives are Pareto free. That is, for such a pair say $(a, b)$ at every profile in the domain there are agents who prefer $b$ to $a$. Note that on forehand we do not expect to know which agents prefer $b$ to $a$. Therefore the agents cannot choose their preferences independently from each other.

To the best of our knowledge these domains have not been studied before. Almost all the "restricted domain" literature considers domains where agents may choose their preferences independently from each other, while here these choices are not free. In Pattanaik and Sen[1969] the domain consists of correlated individual preferences like for instance in Black[1948]. These studies differ considerably from the one here as they aim to find domains allowing for pairwise majority outcomes. Where here we look for domains that allow for anonymous, Pareto optimal and independent of irrelevant alternatives decision rules. Of course, the pairwise majority rule satisfies these conditions, but there are more such rules.

The outcome is rather restrictive. For instance we find that if sufficiently many pairs of distinct alternatives are Pareto free, then the priority rule satisfies these three conditions. The priority rule is based on a given priority order of the alternatives. An outcome on a pair of alternatives, say $(a, b)$, may deviate from the given priority of $b$ over $a$ only if all agents prefer $a$ to $b$. Corollary 1 shows that among the domains restricted by Pareto free pairs only domains allowing for priority rules are domains allowing for Pareto optimal, anonymous and independent of irrelevant alternatives. It also specifies which sets of distinct pairs need to be Pareto free to allow for such rules. Theorem ?? shows that only quasi-priority rules satisfy these three conditions on such domains. Where quasi-priority rules are only marginal deviations of priority rules.

The paper is organized as follows. Section 2 is on the model and notations. Section 3 discusses how decisiveness may spread from some to many pairs of alternatives. Section 4 characterizes all domains restricted by Pareto free pairs allowing for preference rules being Pareto optimal, anonymous and independent of irrelevant alternatives. Section 5 discusses the class of rules satisfying these three conditions on such domains. Section 6 comments on the results.

\section{Pareto free pairs}

For a finite set $S$ let $\# S$ denote its cardinality. Let the finite set $N=\{1,2,3, \ldots, n\}$ denote the set of $n$ agents. The finite set $A=\{1,2, \ldots, m\}$ denotes the set of $m$ alternatives. Alternatives are usually denoted by $a, b, c, x, y$, and $z$.Let $\mathbb{L}$ denote the set of all linear orders on $A$. These are binary relations on $A$ which are weakly complete, asymmetric, and transitive. Preferences are represented by linear orders. Let $\mathbb{P}$ denote the set of all partial orders on $A$. These are binary relations on $A$ which are asymmetric and transitive.

For a partial order $R$ in $\mathbb{P}$ and distinct alternatives $a$ and $b$ it is standard to 
interpret $(a, b) \in R$ as $a$ is (strictly) ordered/preferred above $b$ at $R$. We call $a$ and $b$ twinned by $R$ if for all alternatives $c \in A \backslash\{a, b\}$

$$
\begin{aligned}
(a, c) \in & R \text { if and only if }(b, c) \in R \\
& \text { and } \\
(c, a) \in & R \text { if and only if }(c, b) \in R .
\end{aligned}
$$

In stead of $(a, b) \in R$ we also write $\ldots a \ldots b \ldots=R$. Moreover, $\ldots a b \ldots=R$ means that $a$ is consecutively ordered above $b$ at $R$, that is $(a, b) \in R$ and $a$ and $b$ are twinned by $R$. Notation $a b \ldots=R$ means that $a$ is ordered best and $b$ second best at $R$. Notations for distinct alternatives $a, b$, and $c$ like $\ldots a b c \ldots=R \ldots c a b=R$ and $a b c \ldots=R$ have similar self explanatory interpretations. If both $(a, b) \notin R$ and $(b, a) \notin R$, we call $a$ and $b$ incomparable at $R$. The restriction of $R$ to a non-empty subset $B$ of alternatives is denoted by $\left.R\right|_{B}=\{(x, y) \in B \times B$ : $(x, y) \in R\}$. The reversed order of $R$ is denoted by $-R=\{(y, x):(x, y) \in R\}$.

Combinations of individual preferences are formalized by profiles. A profile $p$ is a function from $N$ to $\mathbb{L}$ assigning to each agent $i$ in $N$ a linear order $p(i)$ in $\mathbb{L}$. That is $p(i)$ is agent $i$ 's preference at profile $p$. Let $\mathbb{L}^{N}$ denote the set of all potential possible profiles. For distinct alternatives $a$ and $b$ and a profile $p$ let $p_{a b}$ denote the number $\#\{i \in N:(a, b) \in p(i)\}$, i.e. the number of agents ordering $a$ above $b$ at profile $p$. A special case occurs when $p_{a b}=n$, as then all agents order $a$ above $b$, meaning that there is unanimity on that pair $(a, b)$. At this case we say a Pareto dominates $b$. For a subset $\mathbb{D}$ of $\mathbb{L}^{N}$ we say that the pair $(a, b)$ is Pareto free if $p_{a b} \neq n$ for all profiles $p$ in $\mathbb{D}$. So, at all profiles in $\mathbb{D}$ alternative $a$ does not Pareto dominate alternative $b$. For all profiles $p$ in $\mathbb{D}$ some agents $i$ in $N$ orders $b$ above $a$. For non-empty subsets $B$ of $A$ we denote the restriction of a profile $p$ to $B$ by $\left.p\right|_{B}$, where $\left.p\right|_{B}(i)=\left.p(i)\right|_{B}$ for all $i \in N$.

Let $P \subseteq A \times A$ denote an irreflexive relation of ordered pairs of alternatives. Let $\mathbb{L}^{N}(P)=\left\{p \in \mathbb{L}^{N}\right.$ : for all $(x, y) \in P$ ordered pair $(x, y)$ is Pareto free at $p\}$. So, $\mathbb{L}^{N}(P)$ denotes that subset of profiles at which all pairs in $P$ are Pareto free. For distinct alternatives $x$ and $y$ let $\min _{x y}=\min _{p \in \mathbb{L}^{N}(P)} p_{x y}$ and $\max _{x y}=\max _{p \in \mathbb{L}^{N}(P)} p_{x y}$ and $\max _{x y}=\max _{p \in \mathbb{L}^{N}(P)} p_{x y}$. As $\min _{x y}+\max _{y x}=$ $n$, depending on whether $(x, y)$ is Pareto free or not we have $\min _{y x}$ equals zero or one and $\max _{x y}$ equals $n$ or $(n-1)$.

Collective decisions are modelled by a preference rule, that is a function $\varphi$ from $\mathbb{L}^{N}(P)$ to $\mathbb{P}$. Such a preference rule assigns to every profile $p$ in $\mathbb{L}^{N}(P)$ a (collective) partial order $\varphi(p)$ of the alternatives. Next we discuss three conditions for preference rules. A preference rule $\varphi$ is said to be

- Pareto optimal, if for all distinct alternatives $x$ and $y$ and all profiles $p$ in $\mathbb{L}^{N}(P)$, with $p_{x y}=n$,

$$
(x, y) \in \varphi(p)
$$

- anonymous, if for all $p \in \mathbb{L}^{N}(P)$ and all permutations $\sigma$ of $N$,

$$
\varphi(p)=\varphi(p \circ \sigma)
$$


- independent of irrelevant alternatives, if for all non-empty subsets $B$ of $A$ and for all $p, q \in \mathbb{L}^{N}(P)$, with $\left.p\right|_{B}=\left.q\right|_{B}$,

$$
\left.\varphi(p)\right|_{B}=\left.\varphi(q)\right|_{B} .
$$

These three conditions are well-known and appear in many studies on Social Choice Theory. In the sequel we say that a preference rule is admissible if it satisfies these three conditions simultaneously. Consider the Pareto rule, $\varphi^{\text {Pareto }}$, defined for an arbitrary profile $p$ in $\mathbb{L}^{N}(P)$ and distinct alternatives $x$ and $y$ as follows

$$
(x, y) \in \varphi^{\text {Pareto }}(p) \text { if (and only if) } p_{x y}=n .
$$

Although the Pareto rule is admissible on every possible domain $\mathbb{L}^{N}(P)$, it is far from resolute. Collectively there exists a preference between two distinct alternatives only if all agents prefer the one above the other. Therefore we study for which domains $\mathbb{L}^{N}(P)$ there exist admissible preference rules that are different from the Pareto rule.

Let $\varphi$ be an admissible preference rule from $\mathbb{L}^{N}(P)$ to $\mathbb{P}$. Note that because of the independence of irrelevant alternatives the outcome of preference rule $\varphi$ can be determined pairwise. That is, for distinct alternatives $x$ and $y$ the collective preference between these two $\left.\varphi(p)\right|_{\{x, y\}}$ is determined by $\left.p\right|_{\{x, y\}}$ the individual preferences between these two. As $\varphi$ is anonymous the number $p_{x y}$ determines the outcome preference between $x$ and $y$ at $\varphi$. So, for each ordered pair of distinct alternatives $x$ and $y$ preference rule $\varphi$ assigns integers $\min _{x y} \leq t \leq \max _{x y}$ such that

$$
p_{x y}=t \Leftrightarrow(x, y) \in \varphi(p) .
$$

Consider $\Phi(x, y)=\left\{t \in\left[\min _{x y}, \max _{x y}\right]_{\mathbb{Z}}: p_{x y}=t \Leftrightarrow(x, y) \in \varphi(p)\right\}$, where $\left[\min _{x y}, \max _{x y}\right]_{\mathbb{Z}}$ denotes the set of integers running from $\min _{x y}$ to $\max _{x y}$. We call $\Phi(x, y)$ the set of winning integers for $(x, y)$. If precisely $t$ agents prefer $x$ to $y$ and $n-t$ agents prefer $y$ to $x$, then $x$ is collectively preferred to $y$ at $\varphi(p)$. Note that $t$ might be a winning integer for $(x, y)$, while $(n-(t+1))$ might be a winning integer for $(y, x)$. Let $\Phi(x, y)^{0}=\Phi(x, y) \backslash\{n\}$ the set of non-trivial winning integers.

Remark 1 Boundary for winning integers

Let $x$ and $y$ be distinct alternatives. If $\min _{x y}=0$, then $\max _{y x}=n$. As $\varphi$ is Pareto optimal we have $n \in \Phi(y, x)$ and therewith $0 \notin \Phi(x, y)$. So, $\Phi(x, y) \subseteq$ $[1, n]_{\mathbb{Z}}$.

If $\varphi$ is different from the Pareto rule, then $\Phi(a, b)^{0}$ is non-empty for some distinct alternatives $a$ and $b$.

\section{Decisiveness}

Proofs on impossibility theorems are often based on an intermediate step saying that decisiveness of a coalition on one single pair of alternatives "spreads" to 
decisiveness of that coalition on all pairs of alternatives. Besides the independence of irrelevant alternatives its deduction is based on the unrestrictedness of the domain and Pareto optimality of the preference rule. Here, consequences of Pareto optimality are limited as pairs might be Pareto free. An approach on this spreading of decisiveness not using Pareto optimality is formulated at Lemma 1 and Lemma 2. At Lemma 3 anonymity is brought in additionally. This results in the absence of strong cycles. That is there cannot be simultaneously a winning integer $s$ on $(a, b)$, a winning integer $t$ is on $(b, c)$ and a winning integer $v$ on $(c, a)$. Based on the exclusion of such strongly winning cycles in the following section, we characterize $P$ such that domains $\mathbb{L}^{N}(P)$ allow for admissible preference rules which differ from the Pareto rule.

For the rest of this section assume that $P \subseteq A \times A$ denotes an irreflexive relation of ordered pairs of alternatives. Let $\varphi$ be a preference rule from $\mathbb{L}^{N}(P)$ to $\mathbb{P}$ which is anonymous, Pareto optimal, and independent of irrelevant alternatives.

Lemma 1 Let $a, b$ and $c$ be distinct alternatives. Let $s \in \Phi(a, b)$ and $t \in$ $\Phi(b, c)$.Then $v \in \Phi(a, c)$ for all $v \in\left[\max \left\{\min _{a c},(s+t-n)\right\}, \min \left\{\max _{a c},(s+\right.\right.$ $t)\}]_{\mathbb{Z}}$.

Proof. Take $v \in\left[\max \left\{\min _{a c},(s+t-n)\right\}, \min \left\{\max _{a c},(s+t)\right\}\right]_{\mathbb{Z}}$. In order to prove that $v \in \Phi(a, c)$ consider subsets $S$ and $T$ of $N$ such that $\# S=s$ and $\# T=t$. As $0 \notin \Phi(a, b)$ and $0 \notin \Phi(b, c)$, we have $S$ and $T$ are both non-empty. We may choose $S$ and $T$ such that $\#(S \cap T)$ is minimal. As $v$ is between $(s+t-n)$ and $(s+t)$, we can choose a subset $V$ of $N$ such that $\# V=v$ and $S \cap T \subseteq V \subseteq S \cup T$. Consider $S \backslash V, V \backslash T, S \cap T, T \backslash S, V \backslash T, N \backslash(S \cup T)$. Note that the union of these six sets is $N$. We distinguish two cases.

Case 1 Only one of these six sets is non-empty. As \# $(S \cap T)$ is minimal and $S$ and $T$ are non-empty, this implies that both $s=n$ and $t=n$. Therewith, $v=n$. As $n=v \leq \min \left\{\max _{a c}, s+t\right\}=\min \left\{\max _{a c}, 2 n\right\}=\max _{a c}$, this means that $\max _{a c}=n$. By Pareto optimality of $\varphi$, this implies that $v=n \in \Phi(a, c)$.

Case 2 At least two of these six sets are non-empty.

Consider the profile $q$ which is defined according to the following table

$$
\begin{aligned}
& c a b \quad \ldots=q(i) \text { for } i \in \quad S \backslash V \\
& \text {.. } a c b=q(i) \text { for } i \in \quad V \backslash T \\
& a b c \quad \ldots=q(i) \text { for } i \in \quad S \cap T \\
& \text {.. bac }=q(i) \text { for } i \in \quad T \backslash S \\
& \text { bca } \ldots=q(i) \text { for } i \in \quad V \backslash T \quad \text { and } \\
& \ldots \quad c b a=q(i) \text { for } i \in N \backslash(s \cup T) \text {. }
\end{aligned}
$$

By reversing the order of the alternatives on the "dots" and switching the order between the alternatives in $\{a, b, c\}$ with those not in $\{a, b, c\}$ we may achieve a profile $r$ like profile $q$ at which all pairs of distinct alternatives in $P$ are Pareto free such that $\left.r\right|_{\{a, b, c\}}=\left.q\right|_{\{a, b, c\}}$. Now as $s \in \Phi(a, b)$ and $t \in \Phi(b, c)$, it follows that $(a, b) \in \varphi(r)$ and $(b, c) \in \varphi(r)$. Transitivity of $\varphi(r)$ implies $(a, c) \in \varphi(r)$. This yields that $v \in \Phi(a, c)$, as $r_{a c}=v$ and $r_{c a}=(n-v)$. 
Lemma 2 Let $a, b, c$ be three distinct alternatives. Let $s \in \Phi(a, b), t \in \Phi(b, c)$, and $v \in \Phi(c, a)$ with $0<s<n$ and $0<t<n$. Then $s \in \Phi(x, y)$ for all distinct alternatives $x, y \in\{a, b, c\}$.

Proof. Note that for distinct alternatives $x$ and $y$ we have $\min _{x y} \leq 1 \leq s \leq$ $n-1 \leq \max _{x y}$ and $\min _{x y} \leq 1 \leq t \leq n-1 \leq \max _{x y}$. So, by applying Lemma 1 we have

1. $s \in \Phi(a, b)$ and $t \in \Phi(b, c)$ implies $s \in \Phi(a, c)$,

2. $v \in \Phi(c, a)$ and $s \in \Phi(a, b)$ implies $s \in \Phi(c, b)$,

3. $t \in \Phi(b, c)$ and $v \in \Phi(c, a)$ implies $t \in \Phi(b, a)$,

4. $t \in \Phi(b, a)$ (by 3) and $s \in \Phi(a, c)$ (by 1) implies $s \in \Phi(b, c)$,

5. $s \in \Phi(b, c)(b y$ 4) and $v \in \Phi(c, a)$ implies $s \in \Phi(b, a)$,

6. $s \in \Phi(c, b)$ (by 2) and $s \in \Phi(b, a)$ (by 5) implies $s \in \Phi(c, a)$.

Now $s \in \Phi(a, b)$, and the conclusions at (1),(2),(4),(5), and (6) complete the proof of this Lemma.

Consider distinct alternatives $x, y$, and $z$. The constellation $s \in \Phi(x, y)$, $t \in \Phi(y, z)$, and $v \in \Phi(z, x)$ is called a winning 3-cycle. If in addition $0<s<n$ and $0<t<n$, then it is called a strongly winning 3-cycle.

Lemma 3 Strongly winning 3-cycle do not exist.

Proof. To the contrary suppose $s \in \Phi(a, b), t \in \Phi(b, c)$, and $v \in \Phi(c, a)$ is a strongly winning 3 -cycle. So, in addition we have $1 \leq s \leq n-1$ and $1 \leq t \leq n-1$. Then Lemma 2 implies that $s \in \Phi(x, y)$ for all distinct $x$ and $y$ in $\{a, b, c\}$. We argue that $v \in \Phi(x, y)$ for all distinct $x$ and $y$ in $\{a, b, c\}$ for all $1 \leq v \leq n-1$.

To prove the latter it is sufficient to prove that

a) if $s \geq 2$, then $(s-1) \in \Phi(x, y)$ for all distinct $x$ and $y$ in $\{a, b, c\}$ and

b) if $s \leq n-2$, then $(s+1) \in \Phi(x, y)$ for all distinct $x$ and $y$ in $\{a, b, c\}$.

Let $s \geq 2$.We have $s \in \Phi(x, y)$ and $s \in \Phi(y, z)$ for distinct alternatives $x, y$, and $z$ in $\{a, b, c\}$. As $1<s<n$, by Lemma 1 we may conclude that $(s-1) \in \Phi(x, z)$. Because $x, y$, and $z$ were chosen arbitrarily it follows that $(s-1) \in \Phi(x, y)$ for all distinct $x$ and $y$ in $\{a, b, c\}$. Which proves $(a)$.

The proof of $(b)$ follows similarly.

So, $[1,(n-1)]_{\mathbb{Z}} \subseteq \Phi(a, b)$ and $[1,(n-1)]_{\mathbb{Z}} \subseteq \Phi(b, a)$. This cannot be as this for instance would mean that 1 is winning on $(a, b)$ and simultaneously $(n-1)$ is winning on $(b, a)$. This contradiction implies that the initial assumption on the existence of strongly winning 3-cycles cannot hold. It therewith proofs the Lemma. 


\section{The character of $P$}

In this section we determine the sets $P$ of Pareto free pairs such that domain $\mathbb{L}^{N}(P)$ allows for admissible preference rules that are different from the Pareto rule.

To derive necessary conditions for $P$ let $\varphi$ be an admissible preference rule from $\mathbb{L}^{N}(P)$ to $\mathbb{P}$ that is different from the Pareto rule. Consider the following weak priority relation $R^{0}=\left\{(x, y) \in A \times A: \Phi(x, y)^{0}\right.$ is non-empty $\}$ on $A$. If $R^{0}$ is asymmetric and transitive it is called partial priority order. As $\varphi$ is different from the Pareto rule we have by Remark 1 that $R^{0}$ is non-empty. Lemma 4 essentially argues that we can find admissible rules on $\mathbb{L}^{N}(P)$ such that $R^{0}$ is asymmetric.

Lemma 4 Let $a$ and $b$ be distinct alternatives such that $(a, b) \in R^{0}$. At an arbitrary profile $p$ and for arbitrary distinct alternatives $x$ and $y$ define

$$
\begin{array}{cccc}
(x, y) \in \bar{\varphi}(p) \quad \text { if } \quad(x, y) \neq(b, a) & \text { and } & (x, y) \in \varphi(p) \\
\text { or if } & (x, y)=(b, a) \quad \text { and } & p_{b a}=n
\end{array} .
$$

Then $\bar{\varphi}$ is an admissible preference rule from $\mathbb{L}^{N}(P)$ to $\mathbb{P}$.

Proof. All in all it is sufficient to prove that $\bar{\varphi}(p)$ yields a transitive relation on $A$. Therefore, suppose that $(x, y) \in \bar{\varphi}(p)$ and $(y, z) \in \bar{\varphi}(p)$. It is sufficient to prove that $(x, z) \in \bar{\varphi}(p)$. Note that if $p_{b a}=n$, then $(b, a) \in \varphi(p)$. So, we may assume that $(x, y) \in \varphi(p)$ and $(y, z) \in \varphi(p)$ and therewith that $(x, z) \in \varphi(p)$. So, if $(b, a) \neq(x, z)$, then by definition $(x, z) \in \bar{\varphi}(p)$. Therefore suppose $(b, a)=$ $(x, z)$. Consequently, $(b, y) \in \varphi(p)$ and $(y, a) \in \varphi(p)$. As $(a, b) \in R^{0}$ there are $1 \leq s \leq(n-1)$ with $s \in \Phi(b, a)$. As $(y, a) \in \varphi(p)$ and $(b, y) \in \varphi(p)$, both $\Phi(y, a)$ and $\Phi(b, y)$ are non-empty. As there are no strong 3 -cycles we have that $\Phi(y, a)=\{n\}$ and $\Phi(b, y)=\{n\}$. Together with $(y, a) \in \varphi(p)$ and $(b, y) \in \varphi(p)$, we may conclude $p_{y a}=n$ and $p_{b y}=n$. But then, $p_{b a}=n$ and by the definition of $\bar{\varphi}$, this yields $(x, z)=(b, a) \in \bar{\varphi}(p)$.

Applying Lemma 4 a number of times yields an admissible preference rule with non-empty asymmetric weak priority relation. Lemma 1 implies that this non-empty weak priority relation is transitive. So, it is a partial priority order. Without loss of generality assume that admissible preference rule $\varphi$ has a corresponding non-empty partial priority order $R^{0}$. Moreover, by Lemma 1 we have that $R^{0}$ transitive with Pareto domination, i.e. for all distinct alternatives $x, y$, and $z$

a) $(x, y) \in R^{0}$ and $(y, z) \notin P$ implies $(x, z) \in R^{0}$, and

b) $(x, y) \notin P$ and $(y, z) \in R^{0}$ implies $(x, z) \in R^{0}$.

All in all by the existence of an admissible preference rule which is different from the Pareto rule we found a non-empty partial priority order that is transitive with Pareto domination. Example 1 shows that such partial priority orders are sufficient to define an admissible preference rule called priority rule. 


\section{Example 1 Priority Rules}

Let $R^{0}$ be a partial priority order which is transitive with Pareto domination. For arbitrary profiles $p$ in $\mathbb{L}^{N}(P)$ and distinct alternatives $x$ and $y$ define the priority rule, $\varphi^{R^{0}}$, as follows

$$
\begin{array}{cccc}
(x, y) \in \varphi^{R^{0}}(p) \quad \text { if } & (x, y) \in R^{0} & \text { and } & p_{x y}>0 \\
\text { or if } & (x, y) \notin R^{0} & \text { and } & p_{x y}=n
\end{array} .
$$

As either $p_{x y}>0$ or $p_{y x}=n$, for distinct alternatives $x$ and $y, \varphi^{R^{0}}(p)$ yields an asymmetric relation on $A$. Also, if $\varphi^{R^{0}}(p)$ is transitive and therewith $\varphi^{R^{0}}$ a preference rule, then the definition above implies that $\varphi^{R^{0}}$ is

- Pareto optimal, as in both cases $p_{x y}=n$ means $(x, y) \in \varphi^{R^{0}}(p)$,

- anonymous, as $(x, y) \in \varphi^{R^{0}}(p)$ is based on numbers of agents preferring $x$ to $y$,

- independence of irrelevant alternatives, as $(x, y) \in \varphi^{R^{0}}(p)$ defined pairwise.

So, it is sufficient to show that $\varphi^{R^{0}}(p)$ is transitive. To that end let $(x, y) \in$ $\varphi^{R^{0}}(p)$ and $(y, z) \in \varphi^{R^{0}}(p)$. It is sufficient to prove that $(x, z) \in \varphi^{R^{0}}(p)$. There are four cases.

Case $(x, y) \in R^{0}$ and $(y, z) \in R^{0}$. As $R^{0}$ is transitive $(x, z) \in R^{0}$. It is sufficient to prove that $p_{x z}>0$. To the contrary suppose $p_{x z}=0$. Then $(z, x) \notin P$ and as $R^{0}$ is transitive with Pareto domination $(z, x) \notin P$ and $(x, y) \in R^{0}$ implies $(z, y) \in R^{0}$. As also $(y, z) \in R^{0}$ this violates the asymmetry of $R^{0}$. So, $p_{x z}>0$ and therewith $(x, z) \in \varphi(p)$.

Case $(x, y) \in R^{0}$ and $(y, z) \notin R^{0}$. So, $p_{x y}>0$ and $p_{y z}=n$. So, $(y, z) \notin P$. As $R^{0}$ is transitive with Pareto domination this yields $(x, z) \in R^{0}$. Also $p_{x y}>0$ and $p_{y z}=n$ by the transitivity of the individual preferences implies $p_{x z}>0$. Hence, by $(x, z) \in R^{0}$ and $p_{x z}>0$ the definition of $\varphi^{R^{0}}(p)$ yields $(x, z) \in \varphi^{R^{0}}(p)$.

Case $(x, y) \notin R^{0}$ and $(y, z) \in R^{0}$. Similarly to the previous case we have $(x, z) \in \varphi^{R^{0}}(p)$.

Case $(x, y) \notin R^{0}$ and $(y, z) \notin R^{0}$. So, $p_{x y}=n$ and $p_{y z}=n$. The transitivity of the individual preferences yields $p_{x z}=n$. Now the definition of $\varphi^{R^{0}}(p)$ yields $(x, z) \in \varphi^{R^{0}}(p)$.

Corollary 1 Let $P \subseteq A \times A$. Let $\mathbb{L}^{N}(P)$ denote the domain of profiles at which all pairs in $P$ are Pareto free. Then (i) and (ii) are equivalent, where

(i) There is a non-empty partial order $R^{0}$ contained in $\mathbb{P}$, that is transitive with Pareto domination,

(ii) There exist preferences rules $\varphi$ from $\mathbb{L}^{N}(P)$ to $\mathbb{P}$, which are simultaneously Pareto optimal, anonymous, and independent of irrelevant alternatives and which are different from the Pareto rule. 
Proof. Example 1 proves (i) implies (ii). Lemma 4 and the arguments between this Lemma and Example 1 prove the existence of such a partial order $R^{0}$. So, (ii) implies (i).

Corollary 1 describes all domains with Pareto free pairs that allow for preference rules being Pareto optimal, anonymous and independent of irrelevant alternatives and being different from the Pareto rule. A description of all such preference rules seems to be rather technical, while the variety of these is relatively small. To show our point and also to demonstrate an additional application of Lemma 3 Example 2 discusses all preference rules that are Pareto optimal, anonymous and independent of irrelevant alternative in case all pairs are Pareto free.

Example 2 All pairs are Pareto free

Let $P=\{(a, b) \in A \times A: a \neq b\}$. So, all pairs are Pareto free. Let $\varphi$ be an admissible preference rule from $\mathbb{L}^{N}(P)$ to $\mathbb{P}$. Let $a$ and $b$ be two distinct alternatives. As all pairs are Pareto free $n \notin \Phi(a, b)$. Therefore, $\Phi(a, b)=$ $\Phi(a, b)^{0} \subseteq[1,(n-1)]_{\mathbb{Z}}$. Note that in general the weak priority relation $R^{0}$ $=\left\{(x, y) \in A \times A: \Phi(x, y)^{0}\right.$ is non-empty $\}$ is not asymmetric. As for any triple of distinct alternatives $x, y$, and $z$, with $(x, y) \in R^{0}$ and $(y, z) \in R^{0}$, Lemma 1 implies that $(x, z) \in R^{0}$, we have that $R^{0}$ is transitive. Further, Lemma 3 implies that $R^{0}$ has no 3 -cycles. Hence, if $(a, b) \in R^{0}$ and $(b, a) \in R^{0}$ then $a$ and $b$ are twinned by $R^{0}$,i.e. for all $c \in A \backslash\{a, b\}$

$$
\begin{aligned}
(a, c) \in & R^{0} \text { if and only if }(b, c) \in R^{0}, \\
(c, a) \in & R^{0} \text { if and only if }(c, b) \in R^{0}, \\
& \text { and } \\
(a, c) \notin & R^{0} \text { or }(c, a) \notin R^{0} .
\end{aligned}
$$

For an arbitrary profile $p \in \mathbb{L}^{N}(P)$ depending on whether $p_{a b} \in \Phi(a, b)$ or not we have $(a, b)$ as a pair in the outcome $\varphi(p)$ or not. So, Lemma 1 and Lemma 3 imply the existence of an irreflexive transitive relation $R$ on $A$ and a mapping $\Phi$ from the pairs in $R$ to the set of non-empty subsets of $[1,(n-1)]_{\mathbb{Z}}$, where

1. distinct alternatives $x$ and $y$ are twinned by $R$, whenever $(x, y) \in R$ and $(y, x) \in R$,

2. $(n-s) \notin \Phi(y, x)$ if $s \in \Phi(x, y)$, whenever $(x, y) \in R$ and $(y, x) \in R$ for distinct alternatives $x$ and $y$,

3. $[\max \{1, s+t-n\}, \min \{(n-1), s+t\}]_{\mathbb{Z}} \subseteq \Phi(x, z)$ for distinct alternatives $x, y$, and $z$, with $(x, y) \in R,(y, z) \in R$, and numbers $s \in \Phi(x, y)$ and $t \in \Phi(y, z)$.

Having such a relation $R$ and mapping $\Phi$, we may determine a preference rule $\varphi^{R, \Phi}$. The outcome at a profile $p \in \mathbb{L}^{N}(P)$ for an arbitrary pair of distinct alternatives, say $x$ and $y$, is defined by

$$
(x, y) \in \varphi^{R, \Phi}(p) \text { if }(x, y) \in R \text { and } p_{x y} \in \Phi(x, y) .
$$


Under the assumption that $\varphi^{R, \Phi}$ is a preference rule, it follows by its definition that the preference rule is admissible. Also, by condition 2 on $R$ and $\Phi$ we have that $\varphi^{R, \Phi}(p)$ is asymmetric. Therefore, in case of all pairs being Pareto free, to show that all rules are characterized by a irreflexive and transitive relation $R$ and a mapping $\Phi$ as spelled out above, it is sufficient to prove that $\varphi^{R, \Phi}(p)$ is transitive. To prove this let $a, b$, and $c$ be distinct alternatives such that $(a, b) \in \varphi^{R, \Phi}(p)$ and $(b, c) \in \varphi^{R, \Phi}(p)$. It is sufficient to prove that $(a, c) \in \varphi^{R, \Phi}(p)$. Consider the following subsets $N_{a b c}, N_{a c b}, N_{c a b}, N_{c b a}, N_{b c a}$, and $N_{b a c}$, where $N_{x y z}=\{i \in N: \ldots x \ldots y \ldots z \ldots=p(i)\}$ for all $\{x, y, z\}=\{a, b, c\}$. Also denote $n_{x y z}=\# N_{x y z}$ for all $\{x, y, z\}=\{a, b, c\}$. Then it is sufficient to show that $(a, c) \in R$ and $\left(n_{a b c}+n_{a c b}+n_{b a c}\right) \in \Phi(a, c)$. Note that by the definition of $\varphi^{R, \Phi}$ yields that $(a, b) \in R$ and $(b, c) \in R$. As $R$ is transitive we have that $(a, c) \in R$. Further, the definition of $\varphi^{R, \Phi}$ implies $\left(n_{a b c}+n_{a c b}+n_{c a b}\right) \in \Phi(a, b)$ and $\left(n_{a b c}+n_{b a c}+n_{b c a}\right) \in \Phi(b, c)$. Now $\left(n_{a b c}+n_{a c b}+n_{c a b}\right)+\left(n_{a b c}+n_{b a c}+n_{b c a}\right)-$ $n=\left(n_{a b c}-n_{c b a}\right)$. So, $\max \left\{1,\left(n_{a b c}+n_{a c b}+n_{c a b}\right)+\left(n_{a b c}+n_{b a c}+n_{b c a}\right)-n\right\} \leq$ $\left(n_{a b c}+n_{a c b}+n_{b a c}\right)$. Also, $\min \left\{(n-1),\left(n_{a b c}+n_{a c b}+n_{c a b}\right)+\left(n_{a b c}+n_{b a c}+n_{b c a}\right)\right\} \geq$ $\left(n_{a b c}+n_{a c b}+n_{b a c}\right)$. Therefore, by condition 3 we have $\left(n_{a b c}+n_{a c b}+n_{b a c}\right) \in$ $\Phi(a, c)$.

Actually, condition 1 is redundant. But it explicates that only on twinned pairs the outcome preferences cat be opposite to each other.

\section{Symmetric $P$ and no globally incomparable pairs}

Corollary 1 describes all domain restrictions based on Pareto free pairs allowing for admissible preference rules different from the Pareto rule. The set of Pareto free pairs is taken arbitrary. In this section we study symmetric sets of Pareto free pair. It seems to be natural to assume that not all agents will simultaneously prefer alternative $x$ above alternative $y$, if they will not simultaneously prefer $y$ above $x$. Therefore in this section we assume $P$ is symmetric, i.e. for distinct alternatives $x$ and $y$

$$
(x, y) \in P \text { implies }(y, x) \in P .
$$

Corollary 1 shows that the priority rule, $\varphi^{R^{0}}$, of Example 1 is an admissible preference rule if there exist admissible rules at all. The priority relation $R^{0}$ may be far from complete and therewith the outcome of the corresponding priority rule. It might even happen that at an admissible preference rule $\varphi$ a pair of distinct alternatives, say $x$ and $y$, is incomparable at every outcome. So, $\Phi(x, y)=\Phi(y, x)=\emptyset$. We call such a pair globally incomparable. To enforce a minimal amount of comparability in the outcomes we will study rules having the following property.

Preference rule $\varphi$ is said to be free of globally incomparable pairs if for all distinct alternatives $x$ and $y$

$$
(\Phi(x, y) \cup \Phi(y, x)) \neq \emptyset .
$$


Let $\varphi$ be an admissible preference rule that is free of globally incomparable pairs. In view of Lemma 4 we may assume that $R^{0}$ is a partial order.

Lemma 5 Let $P$ be a symmetric set of Pareto free pairs. Let $\varphi$ be an admissible preference rule from $\mathbb{L}^{N}(P)$ to $\mathbb{P}$ that is free of globally incomparable pairs such that $R^{0}$ is a partial order. Then $R^{0}$ is negatively transitive, i.e. for all distinct alternatives $x, y$ and $z$

$$
(x, y) \notin R^{0} \text { and }(y, z) \notin R^{0} \text { imply }(x, z) \notin R^{0} .
$$

Proof. Let $(x, y) \notin R^{0}$ and $(y, z) \notin R^{0}$. It is sufficient to prove that $(x, z) \notin R^{0}$. By Corollary 1 we have that $R^{0}$ is transitive with Pareto domination. We distinguish four cases.

Case $1(y, x) \in R^{0}$ and $(z, y) \in R^{0}$. Then by the transitivity of $R^{0}$ we have $(z, x) \in R^{0}$. As $R^{0}$ is asymmetric this yields $(x, z) \notin R^{0}$.

Case $2(y, x) \notin R^{0}$ and $(z, y) \in R^{0}$. Then $(x, y) \notin R^{0}$ and $(y, x) \notin R^{0}$ imply that $\left(\Phi(x, y)^{0} \cup \Phi(y, x)^{0}\right)$ is empty. As $\varphi$ is free from permanently incomparable pairs this implies $(x, y) \notin P$ and $(y, x) \notin P$. But then as $(z, y) \in R^{0}$ and $R^{0}$ is transitive with Pareto domination we have $(z, x) \in R^{0}$. So, the asymmetry of $R^{0}$ implies $(x, z) \notin R^{0}$.

Case $3(y, x) \in R^{0}$ and $(z, y) \notin R^{0}$. This case is similar to Case 2 .

Case $4(y, x) \notin R^{0}$ and $(z, y) \notin R^{0}$. Now if $(x, z) \in R^{0}$, then similarly to Case 2 we would obtain the contradiction $(x, y) \in R^{0}$. So, $(x, z) \notin R^{0}$.

It is basic to see that negative transitivity implies transitivity. Lemma 6 clarifies which negatively transitive relations $R^{0}$ are transitive with Pareto domination.

Lemma 6 Let $R^{0}$ be a partial order on A. Let $P$ be a symmetric set of Pareto free pairs. Then $R^{0}$ is transitive with Pareto domination if and only if for all distinct alternatives $x$ and $y$, with $(x, y) \notin P, x$ and $y$ are twinned by $R^{0}$.

Proof. (if-part) Let all distinct alternatives $x$ and $y$ be twinned by $R^{0}$ whenever $(x, y) \notin P$. We prove that $R^{0}$ is transitive with Pareto domination. Let $x, y, z$ be three distinct alternatives. Let $(x, y) \in R^{0}$ and $(y, z) \notin P$. Without loss of generality it is sufficient to prove that $(x, z) \in R^{0}$. As $(y, z) \notin P$, by the assumption $y$ and $z$ are twinned by $R^{0}$. But then $(x, y) \in R^{0}$ implies $(x, z) \in R^{0}$.

(only if-part) Let $R^{0}$ be transitive with Pareto domination. Let $x, y$ be distinct alternatives that are not twinned by $R^{0}$. It is sufficient to prove that $(x, y) \in P$. As $x$ and $y$ are not twinned by $R^{0}$ without loss of generality we may find an alternative $z$ in $A \backslash\{x, y\}$ such that $(x, z) \in R^{0}$ and $(y, z) \notin R^{0}$. As the assumption $(y, x) \notin P$, together with $(x, z) \in R^{0}$ and $R^{0}$ is transitive with Pareto domination, would imply the contradiction $(y, z) \in R^{0}$, we have $(y, x) \in P$. Because $P$ is symmetric this yields $(x, y) \in P$. 
Theorem 1 Let $P$ be a symmetric set of Pareto free pairs. Then (I) and (II) are equivalent, where

(I) There exist preference rules $\varphi$ from $\mathbb{L}^{N}(P)$ to $\mathbb{P}$, which are simultaneously Pareto optimal, anonymous, independent of irrelevant alternatives and which are free of globally incomparable pairs and which are different from the Pareto rule.

(II) There exist non-empty asymmetric and negatively transitive relations $R^{0}$ on $A$ such that for all distinct alternatives $x$ and $y$

1. $(x, y) \notin P$ implies $x$ and $y$ are twinned by $R^{0}$,

2. $(x, y) \in P$ implies $(x, y) \in R^{0}$ or $(y, x) \in R^{0}$.

Proof. ((I) implies (II)) Let $\varphi$ be a preference rule as described at (I). We prove (II). Because $\varphi$ is not the Pareto rule, it follows that $R^{0}$ is non-empty. In view of Lemma 4 we may assume that $R^{0}$ is a partial order. From Lemma 5 it follows that $R^{0}$ is negatively transitive. Corollary 1 implies that $R^{0}$ is transitive with Pareto domination. Lemma 6 yields (II1). To prove (II2) let $(x, y) \notin R^{0}$ and $(y, x) \notin R^{0}$. We prove that $(x, y) \notin P$ and $(y, x) \notin P$. As $(x, y) \notin R^{0}$ and $(y, x) \notin R^{0}$, we have $\Phi(x, y)^{0}$ and $\Phi(y, x)^{0}$ are empty. As $\varphi$ is free of globally incomparable pairs it follows that $n \in \Phi(x, y) \cup \Phi(y, x)$. So, $(x, y) \notin P$ or $(y, x) \notin P$. By the symmetry of $P$ we now have $(x, y) \notin P$ and $(y, x) \notin P$. This completes the proof of the implication: (I) implies (II).

((II) implies (I)) Suppose $R^{0}$ is an asymmetric and negatively transitive relation on $A$ which satisfies Implications (II1) and (II2). Then $R^{0}$ is transitive because of negative transitivity. Further, Lemma 6 implies $R^{0}$ is transitive with Pareto domination. Example 1 now shows that priority rule $\varphi^{R^{0}}$ is an admissible preference rule from $\mathbb{L}^{N}(P)$ to $\mathbb{P}$. To complete the proof we show that $\varphi^{R^{0}}$ is free of permanently incomparable pairs. This holds by the definition of $\varphi^{R^{0}}$ for distinct alternatives $x$ and $y$ with $R^{0} \cap\{(x, y),(y, x)\} \neq \emptyset$. For distinct alternatives $x$ and $y$ with $R^{0} \cap\{(x, y),(y, x)\}=\emptyset$ condition (II.2) and Pareto optimality guarantee that $x$ and $y$ are not a globally incomparable pairs at $\varphi^{R^{0}}$. This completes the proof.

\section{Some final remarks}

Among the restricted domains studied here Corollary 1 determines precisely those that allow for preference rules which are Pareto optimal,

anonymous, and independent of irrelevant alternatives. Assuming the set of agents is considerably sized, it is most likely to expect that all pairs of distinct alternatives are Pareto free. For instance, in nation wide elections usually potential candidates are only allowed to enter the election if they can certify a certain minimum number of votes. So, the conditions on $P$ spelled out in Corollary 1 are usually satisfied in such elections. Example 2 deals with this special case. In that example all admissible rules are determined. Therewith 
the domain restrictions studied here seem to have some practical relevance as they do avoid the dictatorship outcome of many so-called impossibility theorems. But, the preference rules, which satisfy these three conditions, are almost the opposite of neutral. Indeed as shown in Example 2 the outcome of such preference rules will only marginally differ from a given priority ranking. The preference may only reverse between those pairs of alternatives that are twinned by this priority ranking.

In the following example shows that knowing "more" about the preferences of the agents may help in finding "more" neutral collective decision rules.

Example 3 75\% majority decision

Consider $A=\{1,2,3\}$ is a set of three candidates in a nation wide election. Assume that some reliable sample test shows that in a pairwise comparison the support for 3 against 1 is strictly less than $50 \%$. Then the following pseudo priority rule $\psi$ yields transitive complete and strict orders. At an arbitrary profile $p$ the outcome between $i$ and $j$ is defined as follows

$$
\begin{array}{ccccc}
(i, j) \in \psi(p) \quad \text { if } & j>i & \text { and } & p_{j i}>\frac{3}{4} n \\
\text { or if } & i>j & \text { and } & p_{i j} \geq \frac{1}{4} n
\end{array} .
$$

The outcome is transitive because if at least $75 \%$ of the agents would prefer 2 to 1 and if at least $75 \%$ of the agents would prefer 3 to 2 , then at least $50 \%$ of the agents would prefer 3 to 1 . This, however, is excluded by sample test. Compared to the priority rule we consider this pseudo priority rule more neutral. The decisions against the priority order need a $75 \%$ support instead of a $100 \%$ consensus.

Having a possibility for preference rules immediately raises the question whether this translates to choice rules. Indeed it is straight forward to see that choosing the best ordered alternative of the priority rule yields a choice rule that is Pareto optimal, anonymous and not vulnerable for strategic behavior. So, condition (i) of Corollary 1 is a sufficient condition for a type of domain studied here to allow for such choice rules. However, the following example shows only a weaker condition is needed. As a choice rule is mainly concerned with the top alternatives the "tail" of $P$ is less important.

Example 4 Two best alternatives

Let 1,2 and 3 be three distinct alternatives such that $(x, 1) \in P$ for all $x \geq 3$. For an arbitrary profile $p$ in $\mathbb{L}^{N}(P)$ let the choice function $\kappa$ be defined by

$$
\begin{aligned}
\kappa(p) & =1 \text { if } p_{12}>0 \\
& =2 \text { if } p_{21}=n .
\end{aligned}
$$

Note that if we would have that $P$ is extended like condition (i) of Corollary 1 then $\kappa$ would choose the best alternatives of $\varphi^{R}$ the priority rule based on $R$. Clearly $\kappa$ is anonymous. Also the rule is not vulnerable for strategic behavior 
as it is a monotone "vote" between two alternatives. Next, we will argue that $\kappa$ does not assign alternatives that are Pareto dominated.

Let $\kappa(p)=1$. As $p_{12}>0$ alternative 2 does not Pareto dominate 1 . Further, as $(x, 1) \in P$ for all $x \geq 3$, it holds that $p_{1 x}>0$ for all alternatives $x \geq 3$. So, in that case the outcome is not Pareto dominated.

Let $\kappa(p)=2$. As $p_{21}=n$ alternative 1 does not Pareto dominate 2. Further, as $(x, 1) \in P$ for all $x \geq 3$ we have $p_{1 x}>0$ for all $x \geq 3$. This, the transitivity of the individual preferences, and $p_{21}=n$ imply that $p_{2 x}>0$. Hence, $x \geq 3$ does not Pareto dominate alternative 2. So, in also this case the outcome is not Pareto dominated.

\section{References}

1. Arrow K.J.,1978, Social choice and individual values, Yale University Press (19 $9^{\text {th }}$ edition).

2. Black D., 1948, On the rationale of group decision making, Journal of Political Economy,56, p. 23-34.

3. Gibbard A., 1973, Manipulation of voting schemes: A general result, Econometrica, 41, p. 587-601.

4. Moulin H., 1980, On strategy-proofness and single peakedness, Public Choice, 35, p.437-455.

5. Pattanaik P.K. and Sen A.K., 1969, Necessary and sufficient conditions for rational choice under majority decision, Journal of Economic Theory, 1, p.83-98.

6. Satterthwaite M.A., 1975, Strategy-proofness and Arrow's conditions: Existence and correspondence theorem for voting procedures and social welfare functions, Journal of Economic Theory, 10, p.187-217. 